\title{
Kerf bending: ruled double curved surfaces manufacturing
}

\section{SIGRADI2018 TECHNOPOLITICAS xxii congresso da sociedade iberoamericana de gráfica digital 22th conference of the iberoamerican society of digital graphics 07|08|09|novembro|2018 iau usp | são carlos | sp br}

\author{
Mara Capone \\ UNINA University of Naples Federico II | Italy | mara.capone@unina.it
}

\section{Emanuela Lanzara}

UNINA University of Naples Federico II | Italy | emanuela.lanzara@unina.it

\begin{abstract}
Knowledge of geometric properties of surfaces is crucial for resolution of many manufacturing problems. Developability is an important feature of a surface that allows its manufacture from a flat "strip" of a "flexible" and "non-deformable" material. Digital fabrication technologies and parametric design tools, based on knowledge of geometry, are changing designer way to think. Our research in the field of non-developable surfaces fabrication move from paneling to "kerfing". This technique allows to transform a rigid material in a flexible one. The main problem to solve is how to cut the flat shape to obtain the design surface.
\end{abstract}

Keywords: Non-developable surfaces; Developable surfaces; Shape grammar; Parametric design; Kerfing.

\section{INTRODUCTION}

Knowledge of geometric properties of surfaces is crucial for the resolution of many manufacturing problems. Developability is an important intrinsic property of a surface, because it allows its manufacture starting from a flat "strip" of a "flexible" and "non-deformable" material. Digital fabrication technologies are evolving and becoming more and more widespread. Knowledge of fabrication methods available and parametric design tools, based on geometry, are changing the designer way to think. Advances in this field promotes the experimental use of new materials but also the innovative use of traditional materials, such as wood.

Our research, in the field of wooden curved surfaces fabrication, move from developable surface manufacturing and from "paneling" to "kerfing". This technique consists in transforming a rigid material in a flexible one and the problem to solve is how to cut the flat shape to obtain the design surface. This is a quite simple question to solve for a developable surface but it is a very complex problems to address for double curvature surface manufacturing.

\section{METHODOLOGY}

In our research, we can summarize the methodological approach for wooden curved object manufacturing that moves from theory to practice:

- definition of the theoretical framework, classification of the kerf bending patterns, and analysis of different approaches (patterns uniform distribution vs. patterns optimized distribution);

- experiments for single curvature surfaces manufacturing ( $\mathrm{k}=0$ developable tangential);

- experiments for doubly curvature surfaces manufacturing ( $k<0$ negative curvature);

- experiments for ruled doubly curvature surfaces manufacturing (free form);

- conclusions, analysis of the results and formulations of new hypotheses;

- case study: wooden curved objects manufacturing using kerfing technique.

\section{GEOMETRIC APPROACH}

Geometric genesis of surfaces and knowledge of their properties are crucial for solving many problems, both constructive and measurement. A developable surface can be manufactured starting from a flat "strip", using a flexible and non-deformable material. This is a very important feature of the surface. Geometry studies the properties that don't change and, therefore, the shape of the "strip" to obtain a certain configuration, after a series of rigid movements.

Differential classification of surfaces, introduced by Leonhard Euler (1707-1783) and subsequently used by Monge, allows us to group surfaces according to the definition of curvature, which will be precisely defined by Carl Friedrich Gauss in 1902 (Gauss 1902), in four categories: surfaces with zero curvature, surfaces with positive curvature, surfaces with negative curvature and surfaces with variable curvature.

The curvature of a curve in $P$ is $k$, where $k=1 \backslash r$ and $r$ is the radius of the osculating circle of the curve, we can define the main sections of a surface, the sections of the surface, obtained with planes passing through the normal to the surface in $\mathrm{P}$, with minimum and maximum curvature.
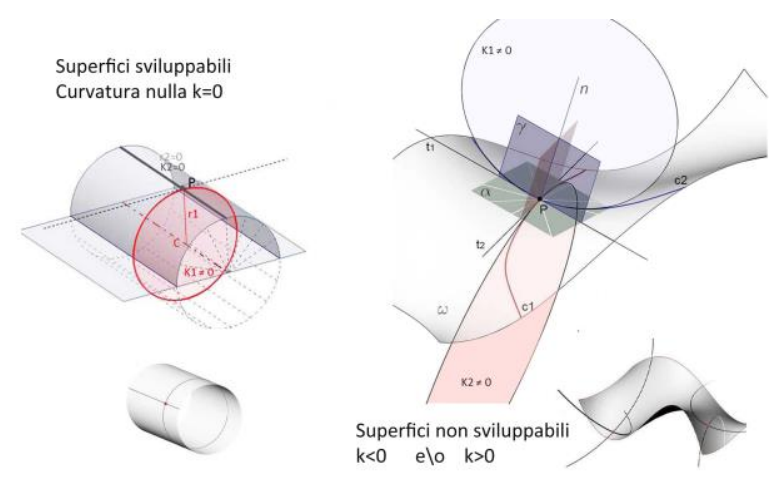

Figure1: Gaussian curvature: osculating circles. Difference between developable $(\mathrm{k}=0)$ and not developable surfaces $(k<0, k>0)$. Source: Authors. 
As we know, Gaussian curvature is the product of the two main curvatures, so it can be positive, negative or equal to zero: it is positive when the osculating circles of the main sections are on the same side of the tangent plane, negative when they are on opposite sides, zero when one of the two main sections is a straight line.

The surfaces with zero curvature are specific ruled surfaces, also called developable (Figure 1).

\section{DEVELOPABLE SURFACES}

A developable surface is a surface for which every generatrix intersects the generatrix infinitely close, then:

- when they intersect on curve c (edge of regression), we have tangent developable;

- when they intersect in a point $\mathrm{V}$ (the edge of regression is a point), we have a conic surface;

- when they intersect at infinity, we have a cylindrical surface.

For this reason, we can group developable surfaces in three groups: conical surfaces, cylindrical surfaces and tangential surfaces. As we know, you can obtain a tangent developable using the tangents to a generic curve $c$, the directrix $c$ is called the edge of regression (Migliari 2009, pp. 213-218).

We can easily generate cylindrical or conical surfaces using a 3D modeling software, extruding the curve in one direction or to a point.

It's more complex generating a tangential developable.

In Descriptive Geometry a tangential developable is generated by motion of tangent line on a spatial curve (the edge of regression). Using algorithmic modeling we have developed a definition to construct developable ruled surfaces using a general spatial curve. This spatial curve can be imported by Rhino or parametrized in relation to specific needs.

Dividing the assigned spatial curve (the edge of regression) in $\mathrm{n}$ parts, our algorithmic definition allows to generate the surface by constructing $\mathrm{n}$ lines (generatrix of the surface) passing through the $\mathrm{n}$ points and tangent to it. It is possible to obtain infinite developable surfaces by modifying the length of the generatrix and the edge of regression. This surface may be cut to define the edge which is otherwise automatically generated as a function of the generatrix length (Figure 3).
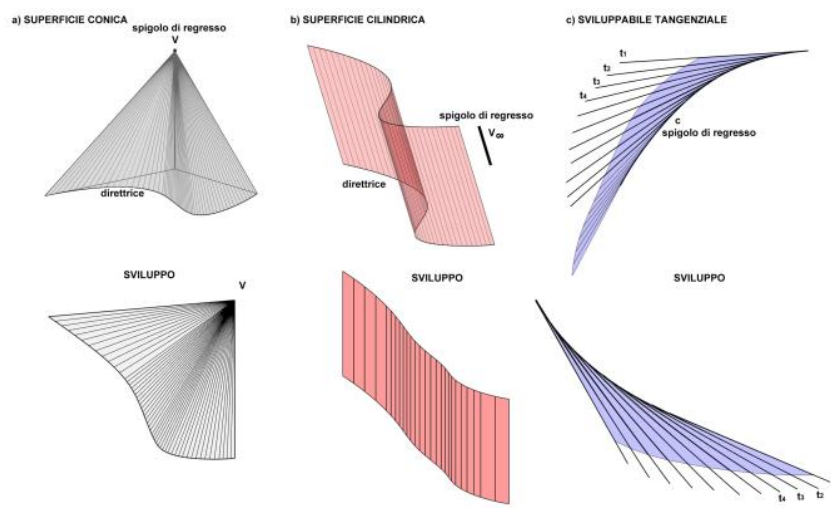

Figure 2: developable surfaces: conical surfaces, cylindrical surface, tangential developable. Source: Authors.
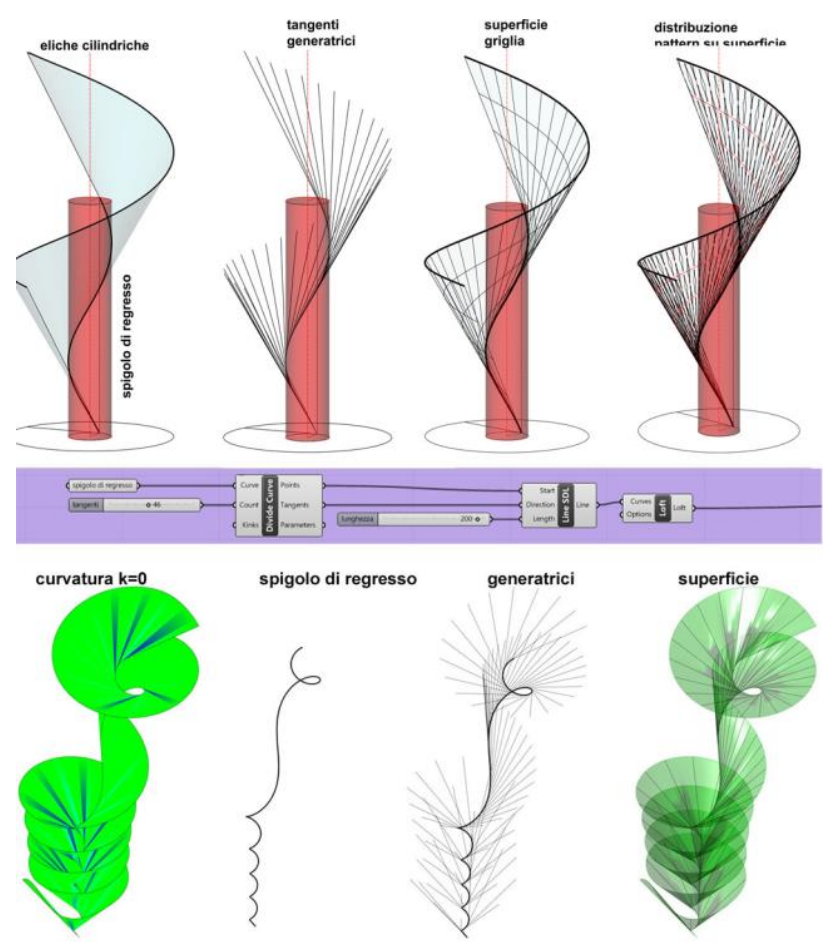

Figure 3: tangential developable: developable helicoid, generic developable. Source: Authors.

A tangential developable specializes if the edge of regression is a cylindrical helix: the surface generated by the motion of a tangent line to a cylindrical helix is a developable helicoid.

The case of the developable helicoid is the simplest, in fact, if the edge of regression is a cylindrical helix, in order to generate the surface we can construct the tangent at a point $\mathrm{P}$ and then make it move along the helix.

In this case, generative modeling is powerful tool, useful not only for reiterating procedures but for verifying theories. In fact, a tangential developable can be unrolled with some unavoidable approximations, as the two consecutive generatrices intersect each others on the edge of regression only in an infinitesimal neighborhood, with $\mathrm{n}$ tending to infinity.

We have realized as a case study two portion of helicoid that can always be developed with the aim of building a wooden lamp.

\section{WHAT 'S THE SHAPE TO CUT?}

One of the main questions to manufacture a $3 D$ surface from a planar panel is to find the 2D shape to cut.

If we have to fabricate a developable surface it is always possible but, if we have to fabricate a complex surface, we have to decide how to determinate its approximate develop. It's very easy to find conical or cylindrical surfaces development using traditional methods or digital tools, on the contrary it is very difficult to find the unrolled shape of a tangent developable surfaces. Using 3D modeling, there is a command that is able to automatically unroll both conical surfaces and cylindrical surfaces. 

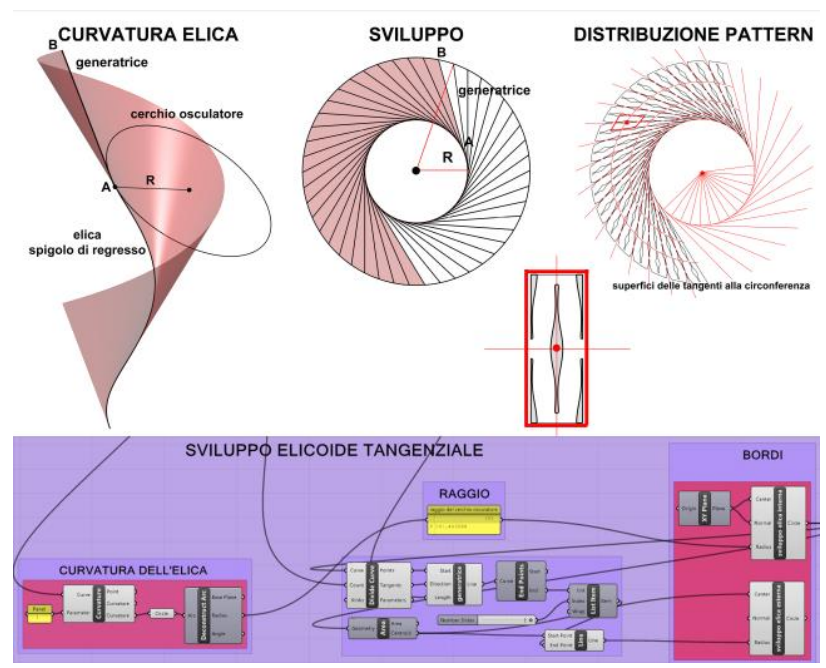

Figure 4: developable helicoid: the shape to cut. Source: Authors.

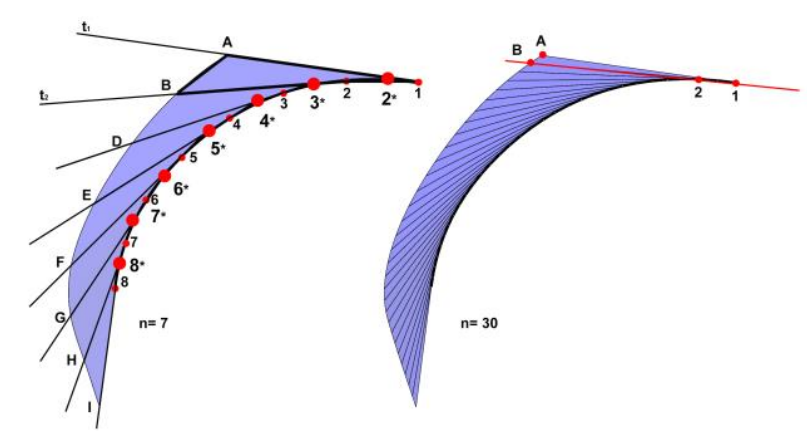

Figure 5: Differential calculus: how to develop a tangential developable. Source: Authors.

The method for finding the unrolled shape of a tangential developable is more complex, in this case differential geometry application is evident.

Monge uses the principles of differential calculus to study the properties of the developable surfaces (Migliari pp. 106-108). Each developable surface can be flattened onto a plane without distortion and, in a limited region, without overlapping.

The unrolled shape of the surface generated by the infinite tangents to a space curve is obtained by considering $n$ generatrices and flattening onto plane the surfaces included between two consecutive generatrices. Two consecutive tangents, $t_{1} t_{2}$, can be considered coplanar if they are very close, then: if we rotate $t_{2} t_{3}$ around $t_{2}$, and we repeat for the following tangents we can find the unrolled surface.

The unrolled surface depends on the edge of regression. Using algorithmic modeling first, we have done a tool that allows to develop a developable helicoid and then we have done any developable tangential.

We know that helixes on developable helicoid turn into concentric circles, for this reason the edge of regression will turn into a circle whose radius depends on $R$, the radius of curvature of the helix. To draw the unrolled shape of the developable helicoid, it's enough to fix the length of the assigned generatrix (for example $A B$ ) on the helix development and drawing a concentric circle with radius OB (Figure 4).
Starting from this theoretical framework and using a generative algorithm in Grasshopper, we have developed a method that allows to find the unrolled shape of any developable tangential.

If we divide the edge of regression into $\mathrm{n}$ parts and we consider $\mathrm{n}$ tangents (generatrices of ruled surface) we have that two consecutive tangents intersect on the edge of regression. This is true only in a small, infinitesimal neighborhood. In fact, if we divide the edge of regression into $n$ parts and we consider two successive tangents, $t_{1}$ and $t_{2}$, from points 1 and 2 , we define the non-flat quadrilateral $A 12 B$. If we extend $B 2$, it intersects the $A 1$ in the point $2^{*}$ (Figure 5 ). Therefore, we can construct the flat triangular face $A 2{ }^{*} B$. In the same way, we extend D3, we have the $B 3^{*} D$ triangle, thus the surface can be divided and approximated in the triangles faces: $A 2{ }^{*} B$, $B 3^{*} D, D 3^{\star} E, D 4^{*} E \ldots$ It is true when $n$ go to infinity, so the point 2 goes to point 1,3 to $2 \ldots$ We can unroll the surface composed by $n$ triangular faces. The approximation of the unrolled surface obviously depends on $\mathrm{n}$. We can evaluate it comparing the metric values of the 3D surface, the length of the edges and the area, with the unrolled shapes that we have constructed (Figure 6).

It may happen that the configuration of the surface is such that portions of unrolled surface overlap with the others, in these cases, it is necessary to divide the design surface into parts in order to manufacturing it.

\section{MANUFACTURING A DOUBLY CURVED SURFACE}

There are two different approaches to define approximate develop of non-developable surface: first we can design the surface and then we can find the flat shape to manufacture it, or we can design 3D surface morphing a planar surface.

We can divide and approximate the 3D surface in order to cover a complex shape by developable strips, which can be unfolded to the plane in an isometric way, without stretching or tearing (Pottmann, 2015). In this way we can fabricate 3D complex shape using materials that can be bent in one direction or rigid material with no possibility of being bent at all.

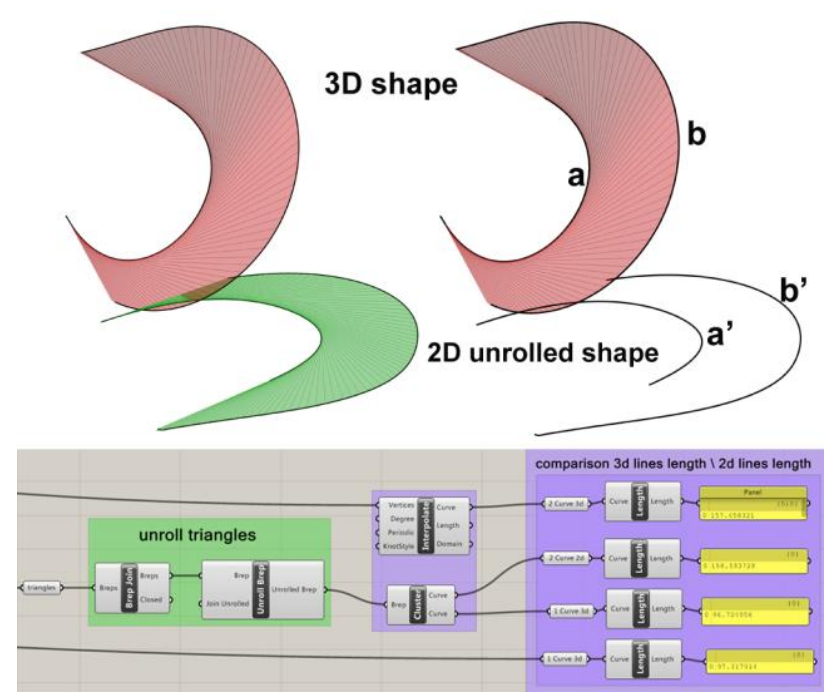

Figure 6: Generative algorithm in Grasshopper to find the unrolled shape of any developable tangential. Source: Authors. 


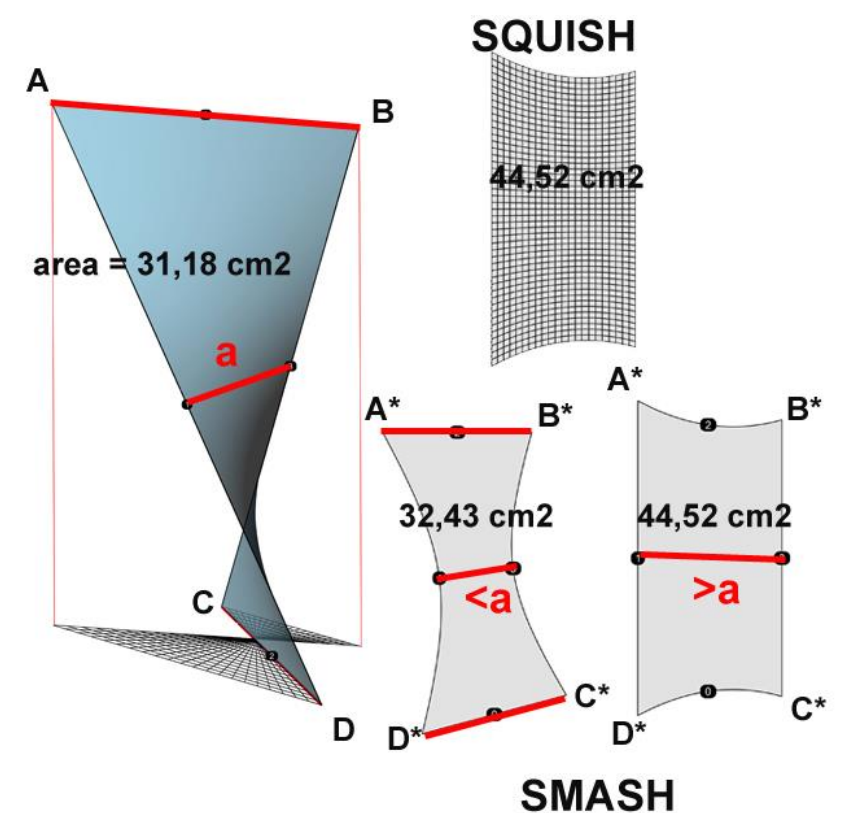

\section{MODELLAZIONE PROCEDURALE "spianamento" - sperimentazione}

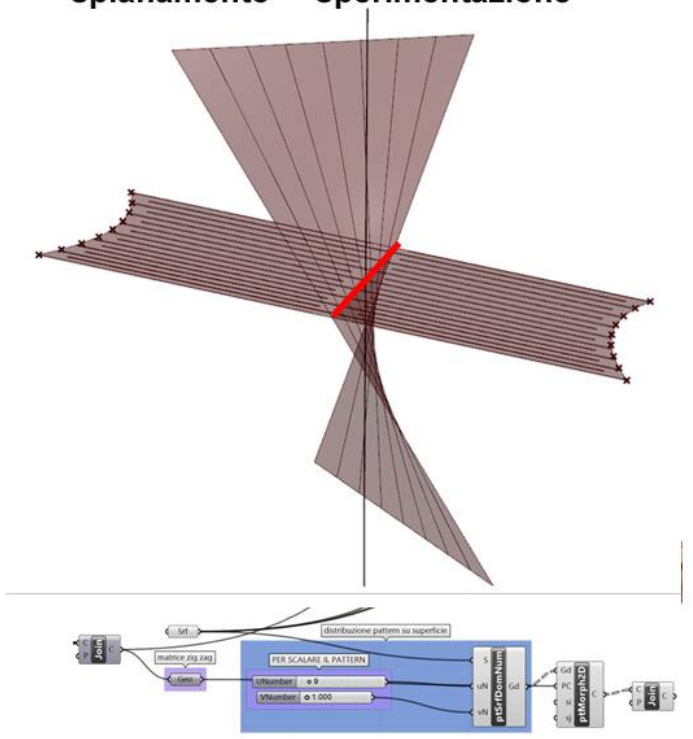

Figure 7: Hyperbolic paraboloid: approximate develop. Source: Authors.

In our research we have considered materials and manufacturing techniques that can solve approximation problems allowing non isometric transformation of the panel thanks to "cuts", kerfing, or "overlapping", bending.

The kerfing technique in addition to making the flexible panel can also make it deformable, this makes it possible to manufacture double curvature surfaces using this technique.

Our tests to unroll the developable helicoid and to unroll a tangential developable is the starting point of our research in progress about manufacturing of non-developable ruled surfaces. Our current experimentation regards nondevelopable surfaces and in particular we are dealing with the problem of identifying the approximate unrolled surface according to the different ways of cutting the panel.

We can find approximate unrolled shape of nondevelopable surfaces useful for certain applications.

We have studied non-developable surfaces and in particular the case of hyperbolic paraboloid, to highlight some of the problems and to define some possible approaches to transform a non-developable surface into a flat surface that, with better approximation, is able to preserve the characteristics of 3D surface.

One of main research goal is to highlight, through the applications, how these approaches can influence the figurative outcome and the manufacturing process.

The hyperbolic paraboloid is a ruled surface that may be generated by a moving straight line that is parallel to a fixed plane. It is a non-developable surface because two consecutive generatrices are always skew lines and Gaussian curvature is always negative.

There are several tools that allow you to automatically obtain the approximate unrolled shape of a non- developable surface: using Rhino the command "smash" and the command "squish".

The critical analysis of the results obtained using 3D modeling software is part of our experimentation.

Using the "smash" command we can automatically generate an approximate unrolled shape for a double curved surface but using this flat shape we can reconstruct the real $3 \mathrm{D}$ shape only if we use a deformable material. The "squish" command uses a different algorithm, performs the smoothing of meshes or 3D NURBS surfaces, modifying the starting area, allowing the display and control of the local compression and stretching zones.

Applying the "smash" and "squish" commands to the hyperbolic paraboloid piece, used in our tests, we obtained different shape (Figure 7). We have done these observations based on results: the area changes compared to the real one and the generatrices of one of the two groups deform themselves. It follows that it will necessarily be breakings and / or overlaps to transform the flatten shape into 3D designed shape. In fact, if the generatrix $A D$ becomes curve, it turns into the curved edge $A^{*} D^{*}$, this must be deformable, therefore the cuts must be made to allow the curve $A^{*} D^{*}$ to assume the configuration straight of the designed shape. Similarly, if the generatrix $A B$ is deformed, it will be necessary to allow that the curve $A^{\prime} B$ is able to transform into the straight segment $A B$ (Figures 7 and 9 ).

We tested different methodologies to simulate deformation according to kerfs made, using generative modeling. Our goal is to identify processes and to develop tools to define the approximate flatten shape of a double curvature based on the knowledge of geometric properties. 

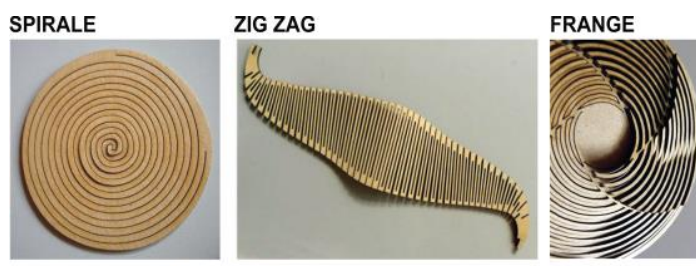

FESSURA
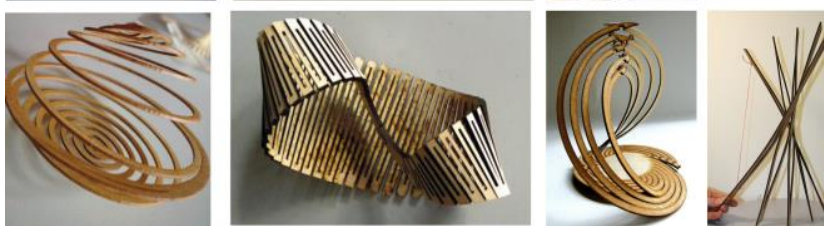

Figure 8: kerfing three different ways of. Source: Authors.

\section{KERFING}

The kerfing technique consists in subtracting material in some points of the panel in order to improve its flexibility. The maximum radius of curvature, that the panel can reach, depends on the material, the panel thickness, the type of kerf and the different distribution ways on the panel in relation to the curvature of the design surface. The main goal of our research was to analyze and to classify the different kerf pattern geometries in relation to the nature of the surfaces and especially to the Gaussian curvature. We've used 2 and $3 \mathrm{~mm}$ ply to make our tests.

We have classified the types of kerf pattern according to three different ways to cut the panel (figure 8):

a) cutting on one side;

b) cutting through the panel;

c) cutting on both sides.

The experimentation is part of a broader research that has involved the study of different kind of "cutting through kerf" in relation to the Gaussian curvature of the designed surfaces.

Our tests show that there are some problems to manufacture double curvature using many of the analyzed patterns.

We have grouped the " cutting through kerf " in four main groups (Munoz 2012): spiral kerf, fringe kerf, zig zag kerf and slit kerf (Figure 8).

1 spiral kerf: it is single or double kerf from the center to the border, it is continuous and it makes the panel flexible in orthogonal direction of the plane. 3D shape depends on the cut line.

2. fringe kerf: a series of kerfs, distributed on the surface according to a geometric rule, come up to the edge and break it. Flexibility depends on kerfs length and geometric rule.

3. zig zag kerf: a series of kerfs that start from the edge of the panel, they are staggered. Flexibility achieved depends on frequency, overlapping of the kerf and on panel thickness. The distance between kerf and the kerf shape is very important for furniture design. Flexibility can be multidirectional.

4. "slit" kerf: they are narrow kerfs that follow a lattice order or pattern. Generally, the plan becomes flexible along the direction of the kerfs and this flexibility can be controlled by providing sectors not affected by kerfs or with different densities. The empty space is very important in this case, because otherwise the contact of the edges is a limit.

First we studied the geometry of the kerf and then we studied the different ways of the pattern distribution on the panel, identifying two different approaches: uniform distribution or optimized distribution (Figure 9).

The studies carried out show that the maximum radius of curvature, that a panel of a given thickness can reach, depends on the type of cut, but also on the pattern distribution on the surface.

Therefore, we have identified some fundamental variables and, through the use of parametric representation tools, we have fabricated a series of prototypes using a slit pattern uniformly distributed on the panel in order to define the type of cut according to the maximum curvature of the designed surface.

The kerfing technique increases the flexibility of the panel by subtracting material and modifying its resistance.

The goal of the most innovative research in this field is to define processes able to find optimized solutions: "cut only where it is needed" to get the designed surface.

We have analyzed some fundamental case studies to understand the different ways of kerfs pattern distribution to optimize surfaces fabrication with variable curvature.

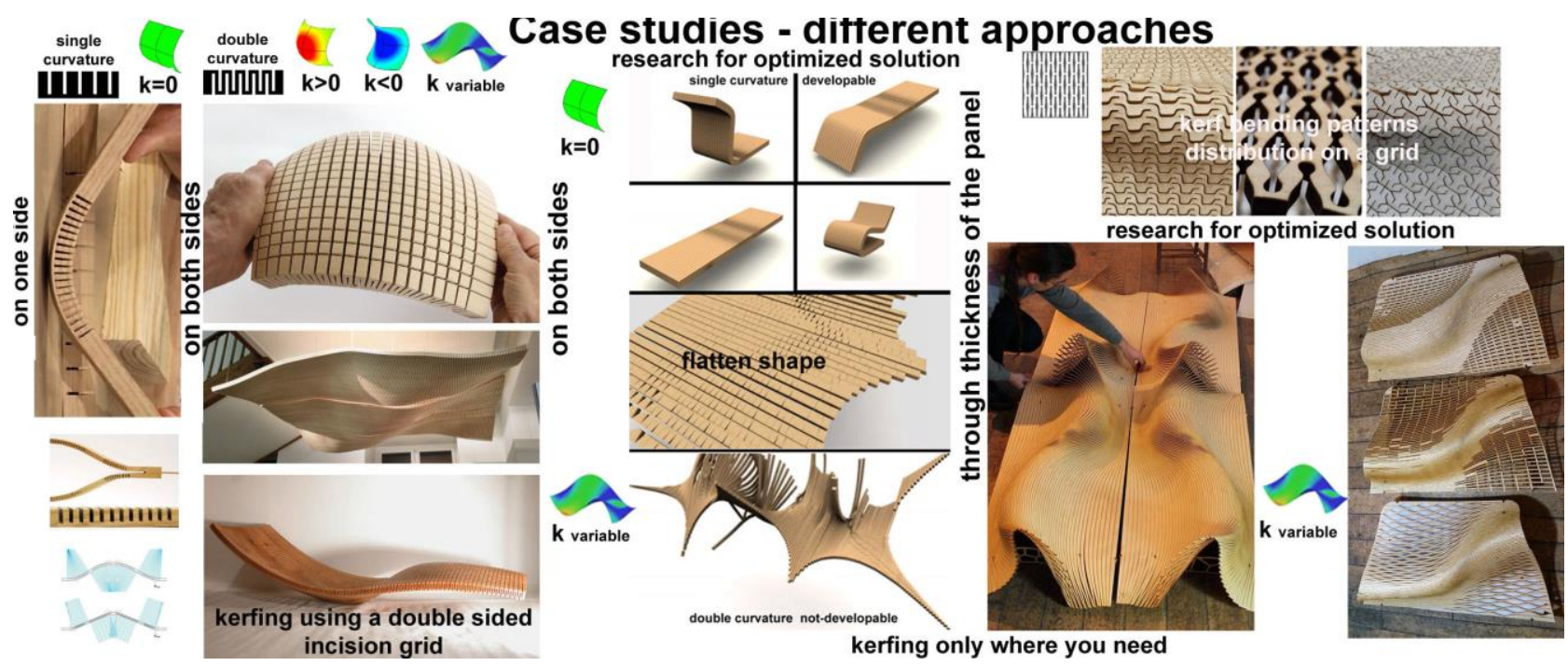

Figure 9: Kerfing: different kind of kerf and different approaches. Source: Authors. 

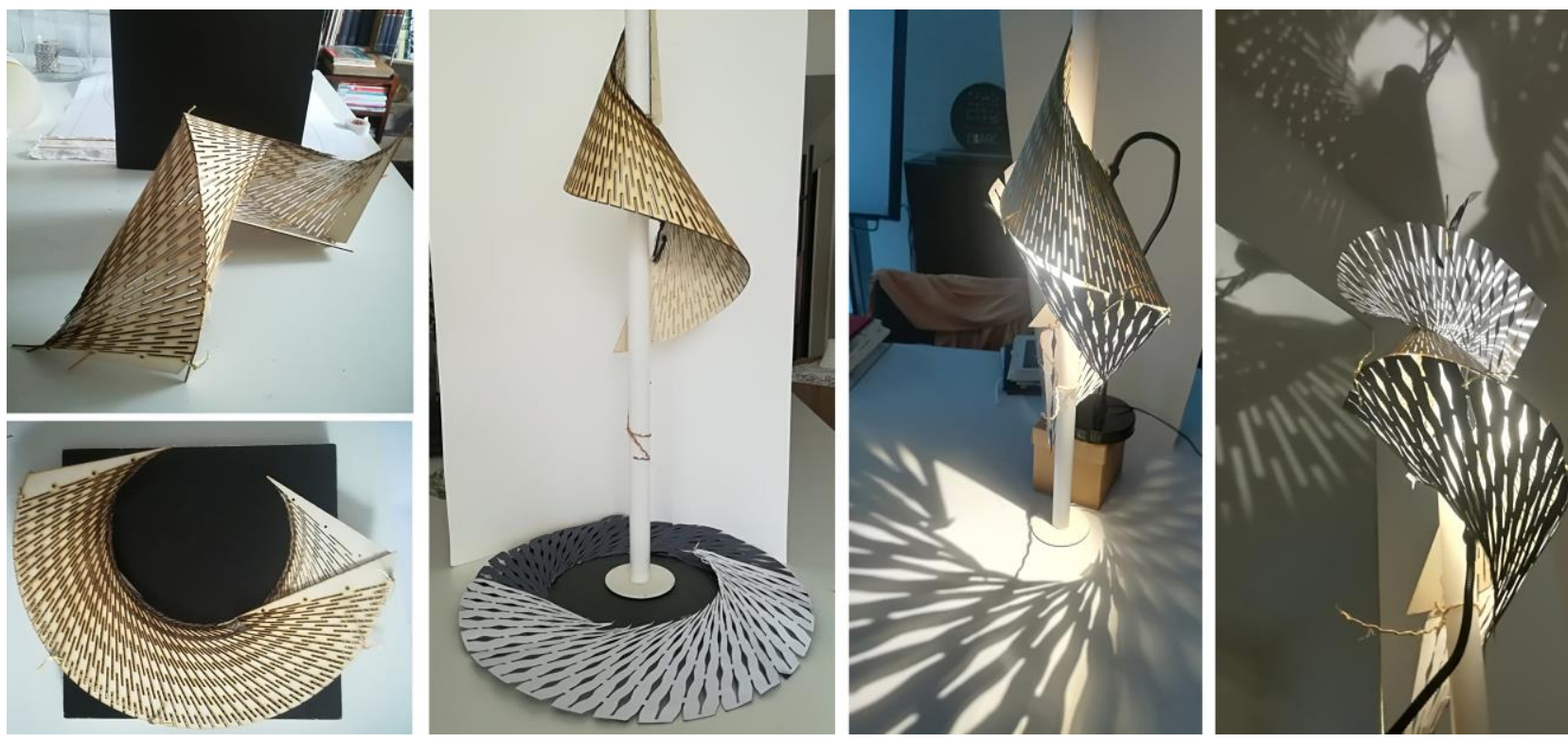

Figure 10: Lamp prototype 1 - developable surface - parts of developable helicoid. Tests manufacturing problems using kerfing technique and shadows design. Source: Authors.

The Wooden Waves, the installation by Mamou-Mani Architects and Buro Happold Engineering, is an emblematic example: the result of experimentation on the slit pattern distributed and sized according to the curvature of the designed surface, that is variable (Figure 9).

\section{PATTERN DISTRIBUTION}

Pattern design process starts from grid layout which has a predefined size. A single geometry is overlapped on intersection points of grid layout as a one- or twodimensional array organization (Güzelci 2016).

Variables like size of pattern units, distance and direction between pattern elements, thickness of the material can be modified to explore the strength of the material.

We tested some pattern using parametric tools and defining assumptions and variables in relation to kerf technology. With regard to this question we have considered the actual machines used for kerfing. In particular, we are analyzing the difference between laser cut and CNC milling, and how they may influence the design process.

We have designed our experiment using $2 \mathrm{~mm}$ fir plywood and $2 \mathrm{~mm}$ MDF, the goal is to know what the maximum bending limit of a material is according to kerf patterns geometry and how to manufacture same 3D shape using material with different thickness.

In our case study manufacturing techniques are used as a design tool. Our aim is to test this methodological path to explore bending behavior of the material using kerfing technique.

The results of our tests show us it is not the subtraction operation itself that increases the bending ability of the surface, but it is lay out pattern distribution that might increase the flexibility. In that way the planar surface begins to perform anisotropic behavior (Güzelci 2016).

We have tested two patterns and their variations to study the relations between patterns geometry and material property and two different kind of kerf pattern, zig zag kerf and slit kerf.
The shape is the start point of our research and not a casual result of trials patterns, among many tests to put on the plane one pattern or mixed patterns to manufacture an assigned doubly curvature surface.

To do that we have produced several prototypes.

We have considered two different approaches:

- pattern distribution on surface using a grid layout on straight line generatrices;

- pattern distribution based on curvature and geometric properties of 3D surface.

In this paper we show two case studies using ruled surfaces:

lamp prototype 1 - developable surface composed by parts of developable helicoid;

lamp prototype 2 - non-developable ruled surface composed by parts of hyperbolic paraboloid.

\section{lamp prototype 1 - developable helicoid}

To distribute the cutting pattern on the 3D surface, we have created a grid using the straight generatrices which we have divided into $\mathrm{m}$ parts (variable) and allow us to modify the grid.

We used Paneling Tools for Grasshopper to generate patterns on curved surfaces. The Paneling Tools "morph $2 \mathrm{D}$ " tool allows you to morph a 2D curve onto a 3D surface. It does this by dividing a surface with a grid. Then it stretches a pattern into the frames created by the grid.

Using algorithms we have distributed the pattern on the surface and on the unrolled surface. In this way we have manufactured the pieces (figure 10).

We are working how to turn our tests into a usable prototype and then into a design product. We are designing how to assemble a complex $3 \mathrm{D}$ object from $2 \mathrm{D}$ pieces, in a simple way. First of all, we are studying the relationship between geometry, kerfs and shadows. We know that the developable helicoid sections by planes orthogonal to cylindrical helix axis are the involutes of the cylinder that contains the helix, therefore we can obtain these shadows up and down of the lamp, on the roof and on the floor (Figure 10). 

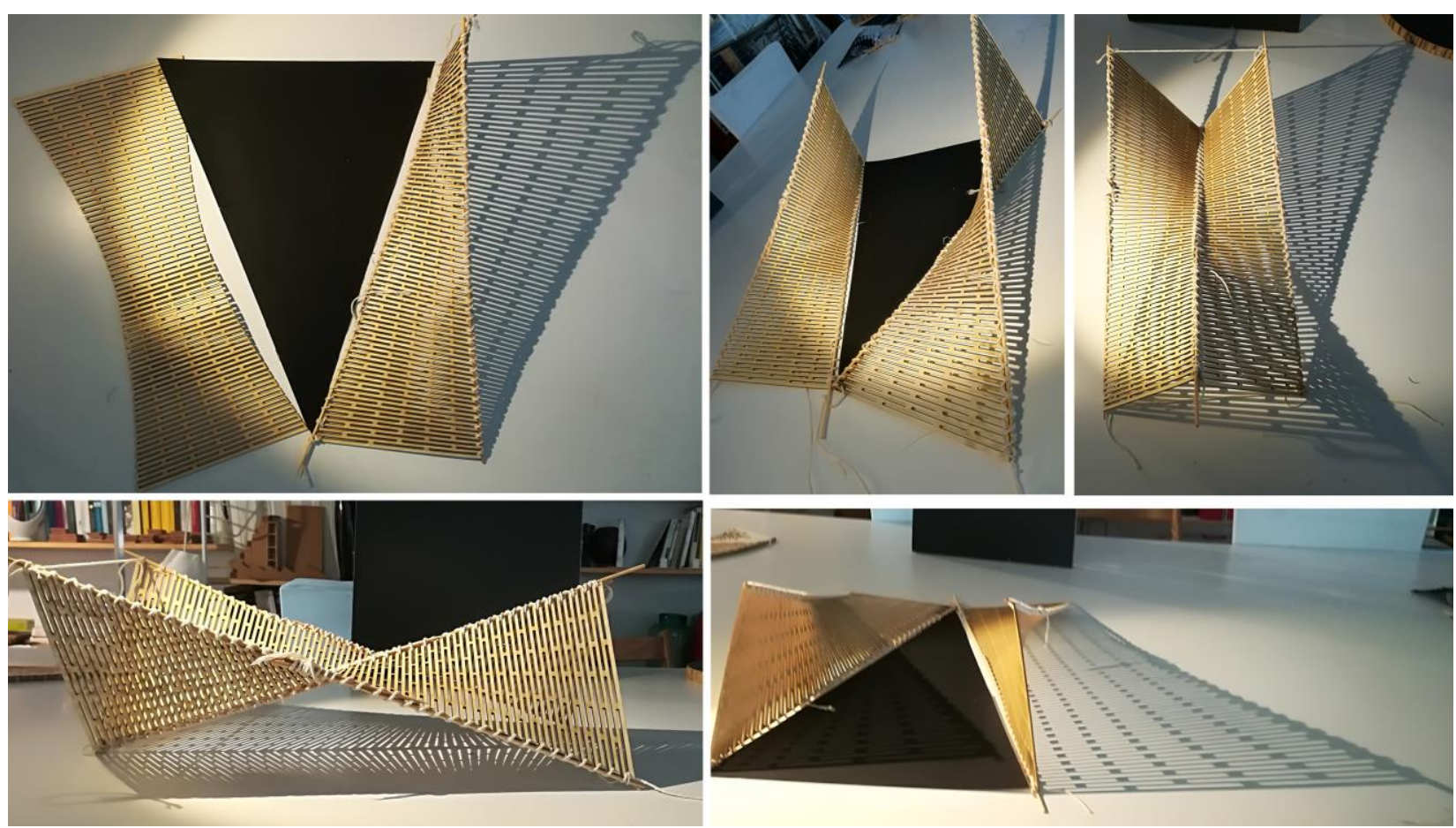

Figure 11: Lamp prototype 2 - non- developable surface - using parts of hyperbolic paraboloid. Approximate develop and pieces shaping. Source: Authors.

We are addressing some problems linked to manufacturing technologies.

\section{lamp prototype 2 - hyperbolic paraboloid}

A hyperbolic paraboloid is a doubly ruled surface generated by motion of a straight generatrix on two straight directrices. The directrices are parallel to the same plane, the first plan-directer, and also the generatrices are parallel to the same plan, the second plan-directer. The main feature of hyperbolic paraboloid is to have two group of straight generatrices.

The hyperbolic paraboloid it isn't developable but, using the "smash" command in Rhino, we can automatically generate two approximate unrolled shapes for a double curved surface. We have used one of these to manufacture our lamp prototype.

We have created a grid using one of generatrices groups and we have distributed slit kerf pattern using Panneling Tools (Figure 11).

Our lamp prototype is composed by four pieces of hyperbolic paraboloid and two elements to connect them. It is very important designing how to connect each piece and how to shape them. It is a very complex work transform the 2D approximate develop into 3D design shape in the case of doubly curved surface.

If you have to bend only in one direction you have only to make flexible the panel. In fact, when you shape a single curvature object you have to force the piece only in one direction, instead, if you have to bend a negative double curvature object you have to bend in two opposite direction, thus there are stresses that tend to return $3 \mathrm{D}$ shape into 2D shape.

Therefore, designing how to connect each piece it is a very hard work.

\section{DISCUSSION}

Kerfing is the act of cutting a series of kerfs (cuts) in a piece of wood in close proximity, so the wood can be curved.

Kerf is defined as the width of material that is removed by a cutting process. It was originally used to describe how much wood was removed by a saw, because the teeth on a saw are bent to the side, so that they remove more material than the width of the saw blade itself, preventing the blade from getting stuck in the wood. When talking about CNC shape cutting with typical cutting processes, kerf is the width of material that the process removes as it cuts through the plate. Over the years some people use the word "kerf" generally referring to cut for bending but now it should only be used when talking about the actual cut width. Each cutting process removes a different amount of material, or kerf. The more precise processes, like waterjet and laser, remove a smaller amount of kerf, which is one of the reasons they can be more precise. In our research we have address the relationship between "kerf" of the laser and the design of our objects.

As a matter of fact that any areas in our design where cut lines come closer than $0.5 \mathrm{~mm}$ together could burn away entirely (Figure 10). Any details narrower than $1 \mathrm{~mm}$ are likely to be very fragile and in some cases can cause the material to warp whilst cutting. As a benchmark, we think that minimum cut widths be no smaller than the corresponding thickness of the material. We can go smaller but this can make our pieces very fragile.

We have corrected our first prototypes of developable helicoid using pattern distribution on our grid in order to respect this rule. 

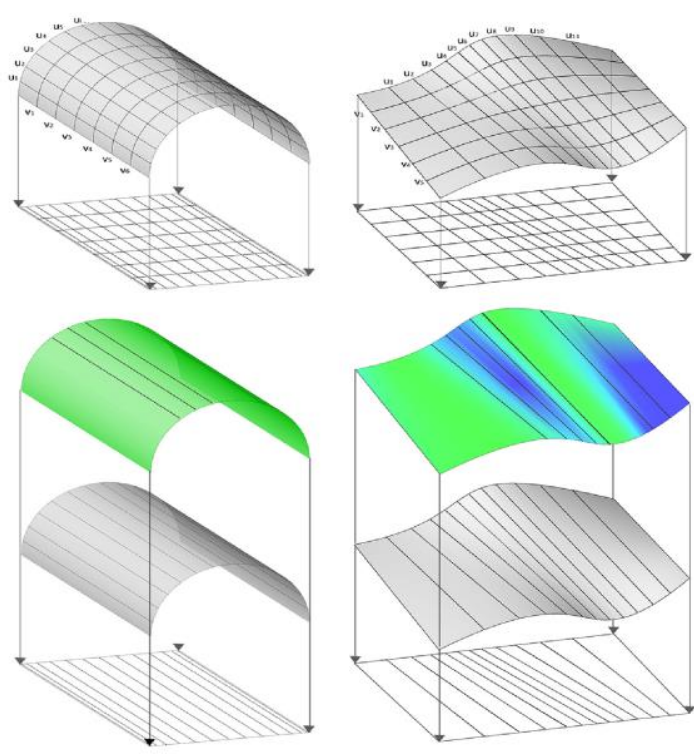
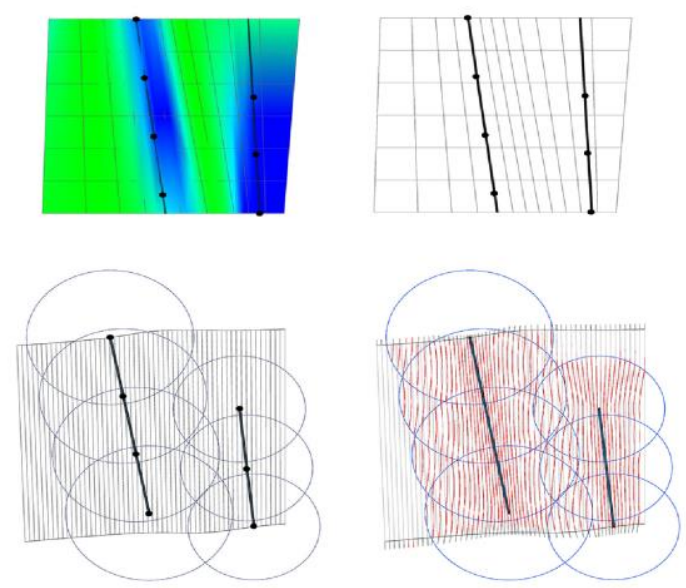

Figure 12: tests for morphing a grid using curvature. Source: Authors.

\section{RESULTS}

The aims of our research are:

- analyzing the relationship between the curvature of surfaces and kerfing techniques;

- testing different ways to optimize the design of kerf bending patterns to make flexible a rigid materials;

- testing different ways to manufacture 3D surface from 2D panel;

To do that we answered these main research questions:

- How do you make wood more flexible?

- How do you manufacture a double curvature surface from a flat panel?

- How do you choose the best pattern for manufacturing your wooden furniture design?

We have fabricated some tests and we are working to manufacture usable prototype made by parts of ruled surface (developable and not-developable).

\section{CONCLUSIONS}

We have presented methods for manufacture design objects using kerfing technique. Our approach is based on geometric knowledge of ruled surface and pattern distribution using a grid on straight generatrices. As we have seen, in the cases of developable surface that we have manufactured, developable helicoid, pattern density automatically achieves the surface curvature.

It isn't true for doubly ruled surfaces. We are studying a technique for applying the pattern based on surface curvature, measuring principle curvature in each direction, then morph a grid along two axis rather than just one. Our method is illustrated by means of different examples, some of which correspond to existing work in kerfing. We have shown how to morph a grid along one axis and how to apply the pattern density to achieve the surface curvature.

The kerfing technique in addition to make flexible panel can also make it deformable, this makes it possible to manufacture double curvature surfaces using this technique. Our tests to unroll the developable helicoid and to unroll a tangential developable is the starting point of our research about manufacturing of non-developable ruled surfaces ( $\mathrm{k}<0$ negative curvature) and the shape to cut.

From our tests we think that the best way for doubly ruled surfaces manufacturing is the slit kerf pattern, distributed on a grid made using the straight generatrices.

Our future studies are in positive curvature surface manufacturing, $k>0$, and in particular we are dealing with the problem of identifying the approximate unrolled surface according to the different ways of cutting the panel.

We are doing tests using a pattern inspired by the works of Ron Resch, and its optimized distribution using more than one direction (Loyola 2017) in relation to Gaussian curvature.

\section{REFERENCES}

Muñoz, P., et al. (2011). Fabricación digital y morfología: la flexibilidad en la generacion de la forma. Ediciones de la forma. Argentina.

Migliari, R. (2009). Geometria descrittiva. CittàStudi, Roma.

Snežana Lawrence, Developable Surfaces: Their History and Application Nexus Network Journal 13 (2011) DOI 10.1007/s00004-011-0087-z; published online 22 November 2011, Kim Williams Books, Turin

Pottmann, H., et al. (2015). Architectural Geometry. Kindle Edition.

Güzelci, O., et al. (2016). Enhancing Flexibility of 2D Planar Materials By Applying Cut Patterns For Hands On Study Models. SIGraDi, Argentina.

Loyola, M., Rozas S., Caldera S., (2017). Kerfing²: a technique for the design, manufacture and optimization of doublecurved elements from rigid wooden plates. SIGraDi, Chile.

Lanzara, Emanuela (2015). Paneling Complex Surfaces. Razionalizzazione di superfici complesse per l'industrializzazione. Phd Thesis. fedoa unina. 